\title{
Simplified equivalent models of large-scale wind power and their application on small-signal stability
}

\author{
Nan DING, Zongxiang LU (ه), Ying QIAO, \\ Yong MIN
}

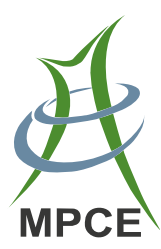

\begin{abstract}
With the rapid growth of grid-connected wind power penetration level, it is necessary to study the impacts of wind power on power system stability. The small-signal stability of power systems with large-scale wind power is explored using the eigenvalue analysis method. A prototype sample system, the two-synchronous-generator system with a wind farm, is proposed for theoretical analysis. Then, simplified models of wind turbines (WTs) and the corresponding equivalent models of wind farms are analyzed. Three kinds of typical WT models, i.e., squirrel cage induction generator, doubly-fed induction generator, and permanent magnet synchronous generator are used. Furthermore, based on the simplified equivalent models, effects of large-scale wind farms on the electromechanical oscillation modes (EOMs) of synchronous systems are discussed. Simulation results indicate that wind farms of the three kinds of WTs have positive effects on EOMs. However, long transmission lines connecting wind farm to the system may produce negative effects on the small-signal stability of the system.
\end{abstract}

Keywords Wind turbine (WT), Small-signal stability, Electromechanical oscillation mode (EOM), Simplified model, Equivalent model

\section{Introduction}

With increasing grid-connected wind power penetration level, wind farms cannot be simply modeled as negative

Received: 31 July 2012/ Accepted: 17 September 2012/Published online: 9 July 2013

(C) The Author(s) 2013. This article is published with open access at Springerlink.com

N. DING, Z. LU, Y. QIAO, Y. MIN, State Key Laboratory of Control and Simulation of Power System and Generation Equipments, Tsinghua University, Beijing 100084, China $(\bowtie)$ e-mail: luzongxiang98@tsinghua.edu.cn load. In addition, the dynamic behaviors of wind turbines (WTs) and its effects of transmission lines need to be considered. Therefore, we discuss the small-signal stability of power systems with large-scale wind power.

Many studies were focused on wind power modeling and small-signal stability analysis, however, there is no consistent conclusion on the problem because of different system models and operating conditions [1]. In general, new rotor angle stability (or called the synchronism stability) problems are not introduced by WTs due to the asynchronous characteristics of squirrel cage induction generator (SCIG), doubly-fed induction generator (DFIG), and the full-scale converter of permanent magnet synchronous generator (PMSG). However, the electromechanical oscillation modes (EOMs) of synchronous systems can be affected by gridconnected wind power. According to [2-4], the effects of large-scale wind farms can be either detrimental or beneficial, depending on many factors, such as wind speed, capacity, and connecting point of wind farms, length of transmission lines connecting wind farms to the system, and original structure of power systems, etc. Thus, the effects of wind farms on EOMs need to be further discovered.

In this paper, we present the eigenvalue analysis method and the prototype sample system for small-signal stability with wind power. Then, the simplified models of three kinds of WTs and the corresponding equivalent model of wind farms can achieve faster calculation speed. Furthermore, based on the simplified equivalent models, the simulation in MATLAB demonstrates the effects of the wind power on EOMs of synchronous systems.

\section{Eigenvalue method and prototype system for small-signal stability}

Small-signal stability is the ability of a power system to maintain synchronism when subjected to small 
disturbances [5]. The disturbance is considered to be small if the equations for describing the resulting response of the system can be linearized. And the eigenvalue analysis is the most critical method in small-signal stability studies.

\subsection{Eigenvalue method}

The dynamic model of the power system can be described by differential algebra equations (DAEs) as:

$$
\left\{\begin{array}{l}
\dot{x}=f(x, y, u), \\
0=g(x, y, u),
\end{array}\right.
$$

where $x, y$, and $u$ are differential state variables, algebra state variables, and control parameter of systems, respectively. During the steady-state, all the derivatives equal zero and the equilibrium points $\left(x_{0}, y_{0}, u_{0}\right)$ can be calculated.

When subjected to small disturbances, (1) can be linearized as (2) and control parameters are considered as constants:

$\left\{\begin{aligned} \Delta \dot{x} & =A \Delta x+B \Delta y, \\ 0 & =C \Delta x+D \Delta y .\end{aligned}\right.$

From the above equation, it can be obtained that

$\Delta \dot{x}=\Lambda \Delta x$.

Defining $\Lambda=A-B D^{-1} C$ as the state matrix, which contains dynamic characteristics of the system. A pair of complex conjugate eigenvalues is $\lambda=\sigma \pm j \omega$, which refers to an oscillation mode. The oscillation frequency $f$ and damping ratio $\xi$ are given by:

$\left\{\begin{array}{l}f=\frac{\omega}{2 \pi}, \\ \xi=\frac{-\sigma}{\sqrt{\sigma^{2}+\omega^{2}}},\end{array}\right.$

where $\xi$ determines the rate of amplitude attenuation of the oscillation.

\subsection{Prototype system}

Electromechanical oscillation mode (EOM) refers to the oscillation mode which is strongly correlated to the rotor speeds and rotor angles of synchronous generators (SGs). The system containing $n$ SGs has $n$-1EOMs. To study the effects of wind power on EOMs of synchronous system, the prototype model must contain at least two SGs. The simplest system model is shown in Fig. 1, and the basic electrical parameters are presented. The base power is $100 \mathrm{MW}$ and each reactance $X$ in Fig. 1 is the sum reactance of the corresponding line and transformer. The dynamic model of SGs is presented in [6], including

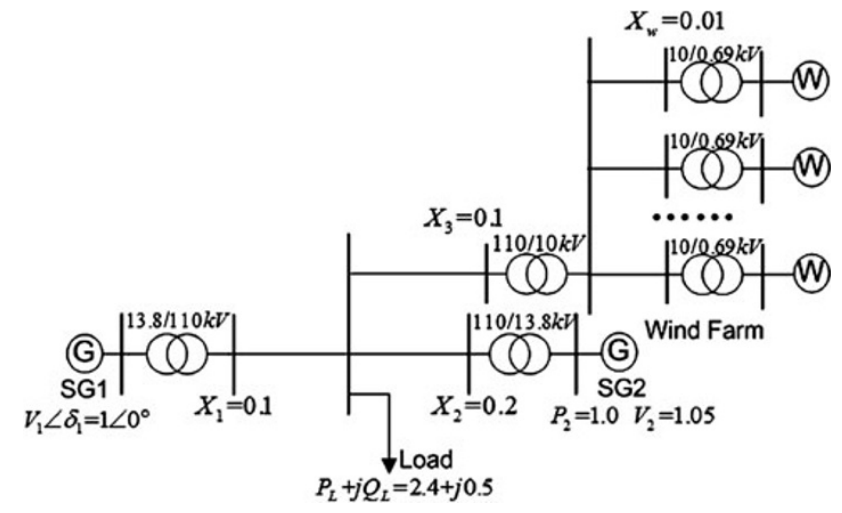

Fig. 1 Model of prototype system

three-order generator model, three-order excitation model, and three-order speed control model. And without wind farm, there is a EOM between the two SGs, whose eigenvalues are $-0.273 \pm \mathrm{j} 9.88$.

\section{Simplified equivalent WT models}

The simplified equivalent models can only be used to study effects of wind power on EOMs of synchronous systems, rather than the stability of WT itself.

\subsection{Detailed WT models}

Detailed mathematical models of three WTs have been built in [7-10], and the model diagrams are shown in Fig. 2. In general, SCIG has six-order dynamic model, and DFIG and PMSG have more than ten-order dynamic models, thus leading to large computational costs in largescale wind power analysis.

\subsection{Simplified WT models}

Some state variables of WTs have significant impacts on EOMs of synchronous system, while others affect it less. To simplify the calculation, the state variables having little relation with EOMs can be ignored.

If a power system lacks damping torque when it subjects to small disturbance, continuous electro-mechanical oscillations will occur. The rotational inertia of SCIG is concentrated in the shaft model, which mainly offers damping torque. Hence, the simplified SCIG model just contains the two-mass shaft model given by (5) to show its effect on EOMs:

$$
\left\{\begin{array}{l}
2 H_{\mathrm{t}} \frac{\mathrm{d} \omega_{\mathrm{t}}}{\mathrm{d} t}=T_{\mathrm{m}}-K \theta_{\mathrm{s}}-D\left(\omega_{\mathrm{t}}-\omega_{\mathrm{r}}\right), \\
2 H_{\mathrm{r}} \frac{\mathrm{d} \omega_{\mathrm{r}}}{\mathrm{d} t}=K \theta_{\mathrm{s}}+D\left(\omega_{\mathrm{t}}-\omega_{\mathrm{r}}\right)-T_{\mathrm{e}}, \\
\frac{\mathrm{d} \theta_{\mathrm{s}}}{\mathrm{d} t}=\omega_{\mathrm{b}}\left(\omega_{\mathrm{t}}-\omega_{\mathrm{r}}\right),
\end{array}\right.
$$


$H_{\mathrm{t}}$ and $H_{\mathrm{r}}$ are the inertia constants of the turbine and generator, respectively; $\omega_{\mathrm{t}}$ and $\omega_{\mathrm{r}}$ are the angle speeds of WT and generator rotor, respectively; $T_{\mathrm{m}}$ is the mechanical torque, $T_{\mathrm{e}}$ is the electromagnetic torque; $\theta_{\mathrm{s}}$ is the shaft twist angle; $K$ is the shaft stiffness coefficient; and $D$ is the damping coefficient.

SCIG and DFIG are both essential asynchronous generators. Thus, although DFIG has many complicated control elements, its behaviors are similar to that of SCIG on EOMs. In this paper, the simplified model of DFIG is the same as that of SCIG given by (5), and the effects of controllers are mainly observed in system load flow.

PMSG has different characteristics compared with SCIG and DFIG. The full-scale converter isolates the generator and the grid, thus only grid-side converter model given by (6) is included in the simplified model

$$
\left\{\begin{aligned}
\frac{\mathrm{d} x_{1}}{\mathrm{~d} t} & =P_{\text {grid_ref }}-P_{\text {grid }}, \\
i_{d \mathrm{~g} \_ \text {ref }} & =K_{\mathrm{p} 1}\left(P_{\text {grid_ref }}-P_{\text {grid }}\right)+K_{\mathrm{i} 1} x_{1}, \\
\frac{\mathrm{d} x_{2}}{\mathrm{~d} t} & =i_{d \mathrm{~g} \_ \text {ref }}-i_{d \mathrm{~g}}, \\
u_{d \mathrm{~g}} & =K_{\mathrm{p} 2}\left(i_{d \mathrm{~g} \_ \text {ref }}-i_{d \mathrm{~g}}\right)+K_{\mathrm{i} 2} x_{2}, \\
\frac{\mathrm{d} x_{3}}{\mathrm{~d} t} & =Q_{\text {grid }}-Q_{\text {grid_ref }}, \\
i_{q \mathrm{~g} \_ \text {ref }} & =K_{\mathrm{p} 3}\left(Q_{\mathrm{grid}}-Q_{\text {grid_ref }}\right)+K_{\mathrm{i} 3} x_{3}, \\
\frac{\mathrm{d} x_{4}}{\mathrm{~d} t} & =i_{q \mathrm{~g} \_ \text {ref }}-i_{q \mathrm{~g}}, \\
u_{q \mathrm{~g}} & =K_{\mathrm{p} 2}\left(i_{q \mathrm{~g} \_ \text {ref }}-i_{q \mathrm{~g}}\right)+K_{\mathrm{i} 2} x_{4},
\end{aligned}\right.
$$

where $K_{\mathrm{p} 1}$ and $K_{\mathrm{i} 1}$ are the proportional and integrating gains of the active power regulator, respectively; $K_{\mathrm{p} 3}$ and $K_{\mathrm{i} 3}$ are the proportional and integrating gains of the reactive power regulator, respectively; $K_{\mathrm{p} 2}$ and $K_{\mathrm{i} 2}$ are the proportional and integrating gains of the grid-side converter current regulator, respectively; and $P_{\text {grid }}$ and $Q_{\text {grid }}$ are the active power and the reactive power of the grid-side converter, respectively; $i_{d \mathrm{~g}}$ and $i_{q \mathrm{~g}}$ where $d$ and $q$ axis currents of the grid-side converter, respectively; and $\mu_{d \mathrm{~g}}$ and $\mu_{q \mathrm{~g}}$ where $d$ and $q$ axis voltages of the grid-side converter, respectively; and the subscript ref means the control reference.

\subsection{Simplified equivalent WT models}

On the basis of simplified models, we use one WT to equivalently represent the whole wind farm in small-signal stability studies to further reduce the computing amount, by extending the WT capacity equal to the output of the whole wind farm.

If all WTs in the wind farm operate at the same point and the WT model is presented by per-unit value, the equivalent WT has the same operating point, and the equivalent transmission line is equal to all transmission lines in parallel.

If all WTs are not at the same operating point, the equivalent operating point needs to be calculated according to the output of the wind farm. The output of equivalent WT yields

$\tilde{P}_{\text {eq }}=\frac{\sum_{i=1}^{n} P_{i}}{\sum_{i=1}^{n} S_{i}}$,

where $P_{i}$ and $S_{i}$ are the actual values of active power and capacity of WT No. $i$, respectively; and $\tilde{P}_{\text {eq }}$ is the per-unit value of active power of equivalent WT. According to $\tilde{P}_{\text {eq }}$, the equivalent operating point can be calculated in the opposite way.

\subsection{Performances of simplified equivalent models}

Figures 3 and 4 show the performances of simplified equivalent WT models at the same operating point. Change tendencies of the prototype system EOM with capacity of wind farms and wind speeds are figured, and only the eigenvalues with positive imaginary part are presented (one EOM corresponds with a pair of complex conjugate eigenvalues). According to (4), the imaginary part of eigenvalue shows the frequency of EOM and the real part shows the damping ratio on the stability of EOM.

The results of detailed, simplified, and simplified equivalent models are shown in Figs. 3 and 4. Since all WTs operate at the same point, the curves of simplified and simplified equivalent models are overlapped. From Figs. 3 and 4 , it can be concluded that the simplified equivalent models can well-present the effects of WTs on EOM of synchronous system, largely reducing the amount of calculations.

For SCIG, the simplified equivalent model leads to slightly conservative results (the results are a little closer to imaginary axis than those of the detailed model). The ignored state variables of SCIG can increase EOM stability, but with a less extent compared with the shaft. Because the dynamic behavior of added controllers in DFIG can decrease the stability of the system to some extent, the stability of EOM with DFIG calculated by the simplified equivalent model is closer to the detailed model than SCIG (the imaginary parts are closer). And as for PMSG, due to the full-scale converter, the curves of detailed, simplified, and simplified equivalent models are all overlapped. Thus, for these three WTs, the simplified equivalent model can well-present the change tendencies of EOM of prototype system with the capacity of wind farm and wind speed.

To test the performances of equivalent model at different operating points, two study cases are designed, there 

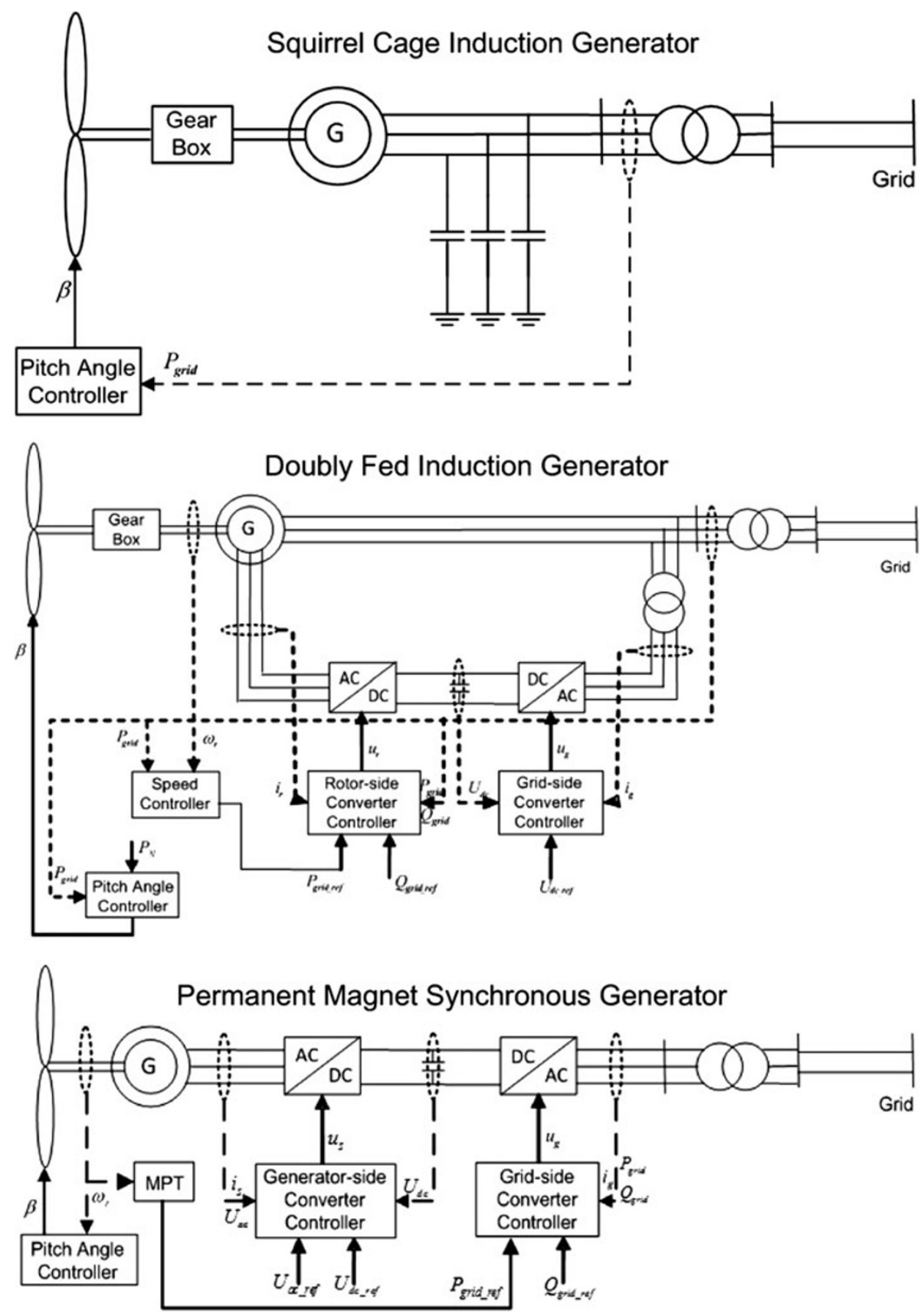

Fig. 2 Model diagrams of three typical wind turbines

are $30 \mathrm{WTs}$ in the wind farm. Case 1: wind speeds of 5, 10 , and 15 WTs are 9,11 , and $13 \mathrm{~m} / \mathrm{s}$. Case 2: wind speeds of 15,10 , and 5 WTs are 5,10 , and $15 \mathrm{~m} / \mathrm{s}$. Table 1 shows the calculation results as well as errors of the real parts which represent the EOM stability. The simplified equivalent WT models also perform well at different operating points, showing the frequency change tendencies and damping ratio of EOM. Thus, the simplified equivalent models can be used in discovering the effects of large-scale wind power on synchronous system EOM.

\section{Effects of wind power on EOMs}

Grid-connected wind power would affect the EOMs of synchronous system because of the dynamic behaviors of WTs and transmission lines. In this section, the effects of 

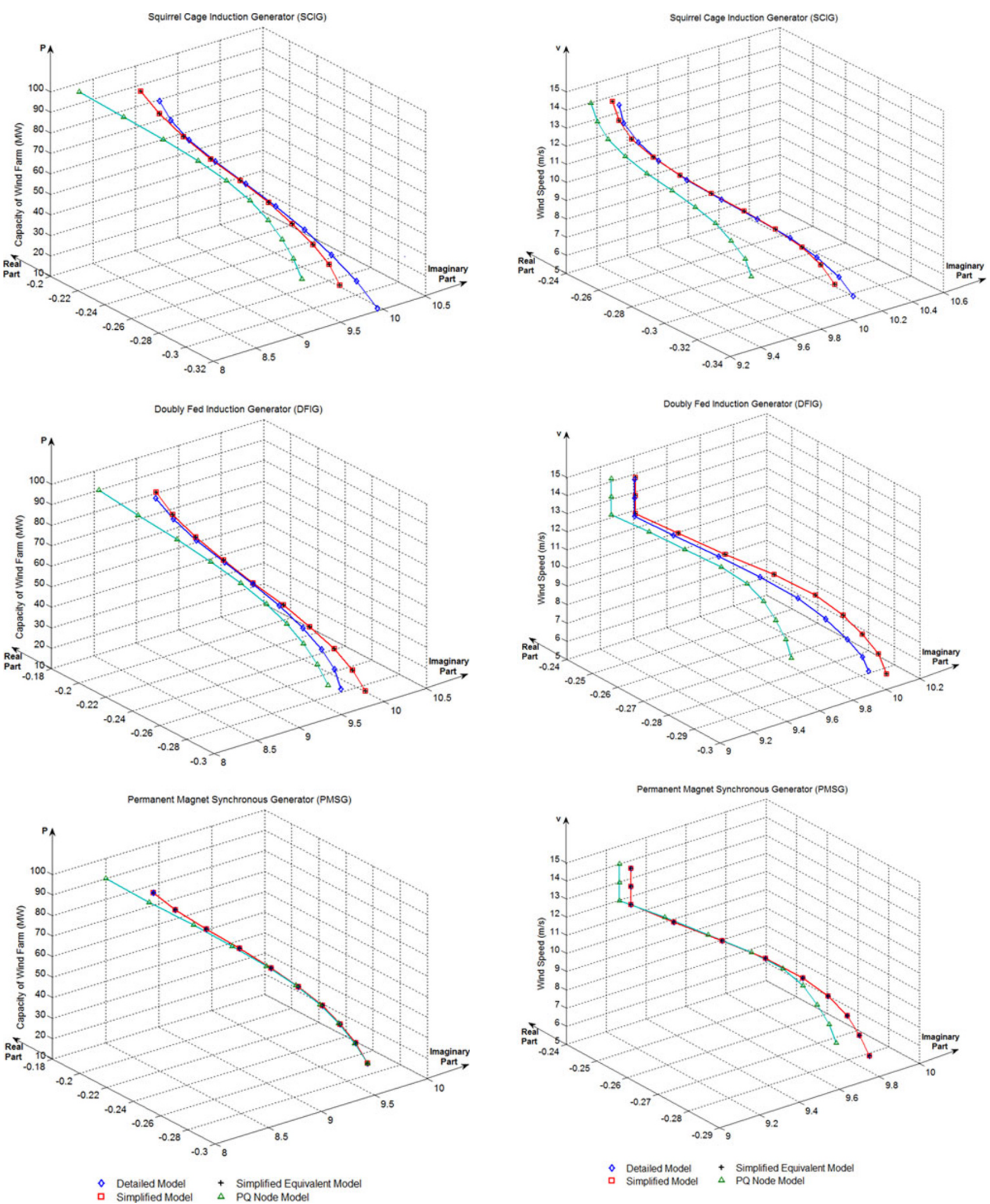

Fig. 3 Change tendency of EOM of prototype system with capacity of wind farm (WTs in full load)

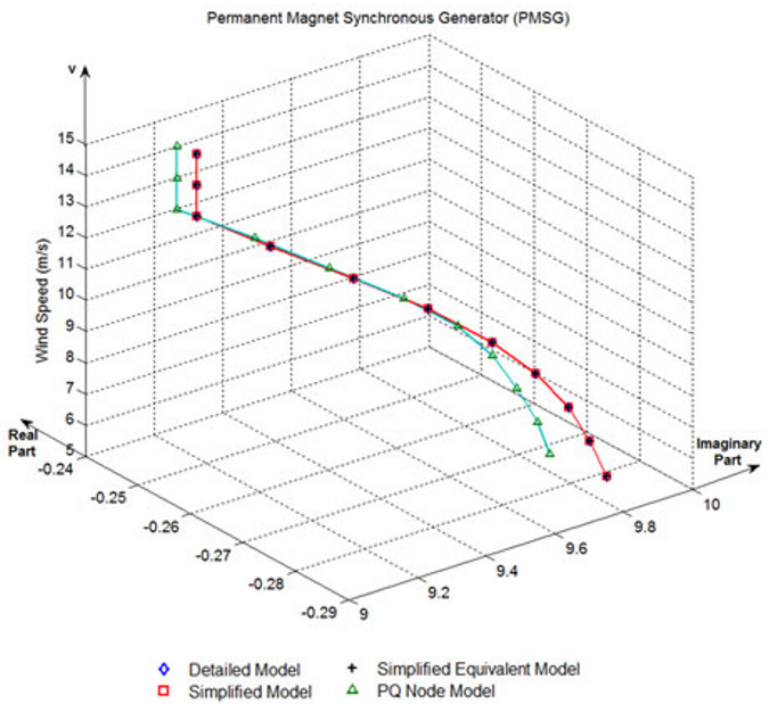

Fig. 4 Change tendency of EOM of prototype system with wind speed (capacity of wind farm $60 \mathrm{MW}$ ) 
Table 1 Performances of simplified equivalent WT models at different operating points (eigenvalues of EOM)

\begin{tabular}{|c|c|c|c|c|c|c|c|c|c|}
\hline \multirow{2}{*}{$\begin{array}{l}\text { Case } \\
\text { no. }\end{array}$} & \multicolumn{3}{|l|}{ SCIG } & \multicolumn{3}{|l|}{ DFIG } & \multicolumn{3}{|l|}{ PMSG } \\
\hline & Detailed model & $\begin{array}{l}\text { Simplified } \\
\text { equivalent } \\
\text { model }\end{array}$ & $\begin{array}{l}\text { Error of } \\
\text { real parts } \\
(\%)\end{array}$ & Detailed model & $\begin{array}{l}\text { Simplified } \\
\text { equivalent } \\
\text { model }\end{array}$ & $\begin{array}{l}\text { Error of } \\
\text { real parts } \\
(\%)\end{array}$ & Detailed model & $\begin{array}{l}\text { Simplified } \\
\text { equivalent } \\
\text { model }\end{array}$ & $\begin{array}{l}\text { Error of } \\
\text { real parts } \\
(\%)\end{array}$ \\
\hline 1 & $-0.276 \pm j 9.53$ & $-0.271 \pm j 9.53$ & 1.8 & $-0.260 \pm j 9.44$ & $-0.261 \pm j 9.48$ & 0.4 & $-0.258 \pm j 9.38$ & $-0.258 \pm j 9.38$ & 0 \\
\hline 2 & $-0.303 \pm j 9.95$ & $-0.295 \pm j 9.97$ & 2.6 & $-0.280 \pm j 9.81$ & $-0.281 \pm j 9.90$ & 0.4 & $-0.272 \pm j 9.73$ & $-0.272 \pm j 9.73$ & 0 \\
\hline
\end{tabular}

wind power are discussed based on the simplified equivalent models.

\subsection{Influences of WTs}

To highlight the influences of dynamic behaviors of WTs, eigenvalue results based on simplified equivalent WT models are compared with the results based on constant PQ node models, namely, treating the wind farm as constant PQ node without dynamic behaviors. Simulation results are also shown in Figs. 3 and 4. The results of PQ node models show the effects of change in load flow on EOM with wind power, while the errors of simplified equivalent models and PQ node models reveal the effects of WTs dynamic behaviors.

Because SCIG and DFIG are asynchronous in characteristics, they can ameliorate the stability of EOM (the results of simplified equivalent models are farther from imaginary axis than those of PQ node models). And due to the full-scale converter, PMSG has little effect on EOM, but can still improve its stability. On the basis of previous analysis, it is acknowledged that without wind farms, the eigenvalues of EOM between the two SGs are $-0.273 \pm j 9.88$. Figures 3 and 4 show that the damping ratio of EOM with wind power is lower than the one without wind power. The results of $\mathrm{PQ}$ node models demonstrate that the change in load flow is the main reason for the decrease of stability rather than dynamic behaviors of WTs.

In addition, for all these three WTs, lower wind speed has better EOM stability. At a lower speed, the asynchronous characteristics are dominant in SCIG and DFIG, and the speed and the power control of DFIG and PMSG becomes easier (Table 1).

\subsection{Influences of transmission lines on EOMs}

The transmission lines of the wind farm can also affect the load flow of the system and EOM. In Fig. $1 X_{3}$ is the sum reactance of the wind farm transformer and transmission line.

Figure 5 shows the change tendency of EOM of prototype system with the longer transmission line. The longer transmission lines may lead to less EOMs stability. In fact, because regions with abundant wind resources are usually

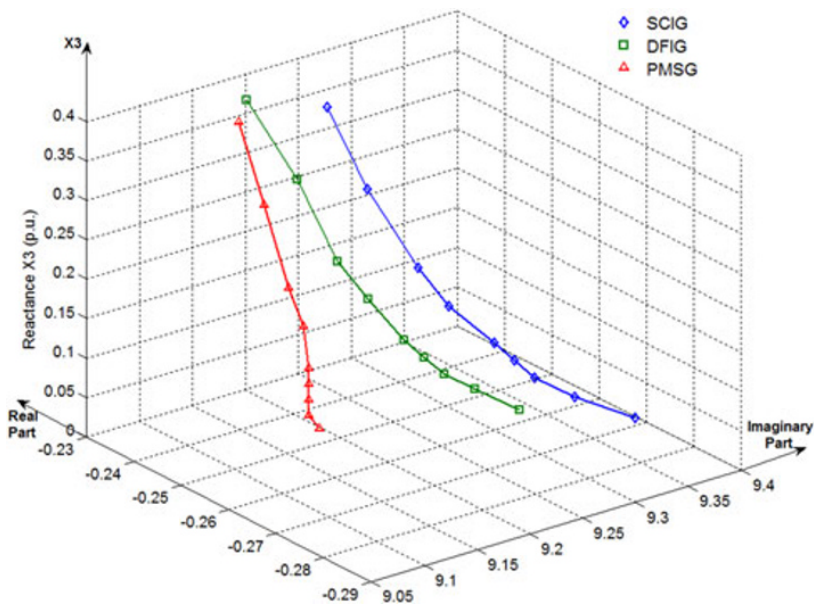

Fig. 5 Influences of transmission lines on EOM of prototype system (capacity of wind farm $60 \mathrm{MW}$ )

far from load centers, long transmission lines are unavoidable for large-scale wind power. Thus, we pay more attention to the impacts of long transmission lines on small-signal stability.

\section{Conclusion}

The simplest system model is used for discovering the effects of large-scale wind power on EOMs of synchronous system. The prototype model contains two SGs which can produce one EOM, and one wind farm is paralleled in the system.

The simplified equivalent models of three WTs are used for analyzing small-signal stability.

The simplified WT model of SCIG and DFIG contains two-mass shaft model, and the simplified model of PMSG contains a full-scale converter model. The equivalent model is employed to represent the wind farm by extending the output power of equivalent WT until it equals the whole wind farm. Simulation results show that the simplified equivalent model is suitable for representing the effects of large-scale wind power on EOMs, no matter the operating points of WTs are the same or not.

The EOMs of synchronous system can be affected by large-scale grid-connected wind farms. Simulation results 
show that the dynamic behaviors of these three WTs have positive effects on EOM, improving the stability of EOM. Furthermore, the lower wind speed usually indicates a better EOM damp.

However, transmission lines will have adverse effects. Since most large-scale wind farms are far from load centers, long transmission lines need wind power integration. And they usually produce a negative effect on EOM, decreasing the small-signal stability of the system.

Acknowledgments This work is supported by State Key Laboratory of Control and Simulation of Power System and Generation Equipments, Tsinghua University; National Natural Science Foundation of China (51190101); National High-tech R\&D Program (863 Program) (2011AA05A104); National Natural Science Foundation of China (51077078).

Open Access This article is distributed under the terms of the Creative Commons Attribution License which permits any use, distribution, and reproduction in any medium, provided the original author(s) and the source are credited.

\section{References}

[1] Sun Y, Wang L, Li G et al (2010) A review on analysis and control of small signal stability of power systems with large scale integration of wind power. In: Proceedings of the 2010 international conference on power system technology: technological innovations making power grid smarter (POWERCON'10), Hangzhou, 24-28 Oct 2010, p 6

[2] Gautam D, Vittal V, Harbour T (2009) Impact of increased penetration of DFIG-based wind turbine generators on transient and small signal stability of power systems. IEEE Trans Power Syst 24(3):1426-1434

[3] Mendonca A, Lopes JAP (2005) Impact of large scale wind power integration on small signal stability. In: Proceedings of the 2005 international conference on future power systems (FPS'05), Amsterdam, 16-18 Nov 2005, p 5

[4] Knüppel T, Nielsen JN, Jensen KH et al (2012) Small-signal stability of wind power system with full-load converter interfaced wind turbines. IET Renew Power Gener 6(2):79-91

[5] Kundur P, Paserba J, Ajjarapu V et al (2004) Definition and classification of power system stability. IEEE Trans Power Syst 19(3):1387-1401
[6] Ni Y, Chen S, Zhang B (2002) Theory and analysis of dynamic power system. Tsinghua University Press, Beijing (in Chinese)

[7] Lopez YU, Navarro JAD (2008) Small signal stability analysis of wind turbines with squirrel cage induction generators. In: Proceedings of the 2008 IEEE/PES transmission and distribution conference and exposition: Latin America, Bogota, 13-15 Aug 2008, p 10

[8] Wu F, Zhang XP, Godfrey K et al (2007) Small signal stability analysis and optimal control of a wind turbine with doubly fed induction generator. IET Gener Transm Dis 1(5):751-760

[9] Hansen AD, Jauch C, Sørensen P et al (2003) Dynamic wind turbine models in power system simulation tool DIgSILENT. Ris $\varnothing-\mathrm{R}-1$, Ris $\varnothing$ National Laboratory, Roskilde

[10] Margaris ID, Hatziargyriou ND (2010) Direct drive synchronous generator wind turbine models for power system studies. In: Proceedings of the 7th Mediterranean conference and exhibition on power generation, transmission, distribution and energy conversion (MedPower'10), Agia Napa, 7-10 Nov 2010, p 7

\section{Author Biographies}

Nan DING received the B.S. degree from Tsinghua University, Beijing, China, in 2011. She is now a master student in Department of Electrical Engineering, Tsinghua University. Her research interest is small-signal stability with wind power.

Zongxiang LU received the B.S. and Ph.D. degrees from Tsinghua University, Beijing, China, in 1998 and 2002, respectively. He is now an associate professor in Department of Electrical Engineering, Tsinghua University. His research interests include power system reliability, large-scale variable generation integration, DG and microgrid.

Ying QIAO received the B.S. and Ph.D. degrees from Shanghai Jiaotong University, Shanghai, China, and Tsinghua University, Beijing, China, in 2002 and 2008, respectively. She is now a lecturer in Department of Electrical Engineering, Tsinghua University. Her research interests include renewable energy and power system security and control.

Yong MIN received the B.S. and Ph.D. degrees from Tsinghua University, Beijing, China, in 1984 and 1990, respectively. He is now a professor in Department of Electrical Engineering, Tsinghua University. His research interests include Power System Security, Stability, and Control. 